\title{
Global Asymptotic Stability of a Family of Nonlinear Difference Equations
}

\author{
Maoxin Liao \\ School of Mathematics and Physics, University of South China, Hengyang, Hunan 421001, China \\ Correspondence should be addressed to Maoxin Liao; mxliao@163.com \\ Received 17 June 2013; Accepted 9 November 2013 \\ Academic Editor: Cengiz Çinar \\ Copyright (C) 2013 Maoxin Liao. This is an open access article distributed under the Creative Commons Attribution License, which \\ permits unrestricted use, distribution, and reproduction in any medium, provided the original work is properly cited. \\ In this note, we consider global asymptotic stability of the following nonlinear difference equation $x_{n}=\left(\prod_{i=1}^{v}\left(x_{n-k_{i}}^{\beta_{i}}+1\right)+\prod_{i=1}^{v}\left(x_{n-k_{i}}^{\beta_{i}}-\right.\right.$ \\ $1)) /\left(\prod_{i=1}^{v}\left(x_{n-k_{i}}^{\beta_{i}}+1\right)-\prod_{i=1}^{v}\left(x_{n-k_{i}}^{\beta_{i}}-1\right)\right), n=0,1, \ldots$, where $k_{i} \in \mathbb{N}(i=1,2, \ldots, v), v \geq 2, \beta_{1} \in[-1,1], \beta_{2}, \beta_{3}, \ldots, \beta_{v} \in(-\infty,+\infty)$, \\ $x_{-m}, x_{-m+1}, \ldots, x_{-1} \in(0, \infty)$, and $m=\max _{1<i<v}\left\{k_{i}\right\}$. Our result generalizes the corresponding results in the recent literature and \\ simultaneously conforms to a conjecture in the work by Berenhaut et al. (2007).
}

\section{Introduction}

The study of dynamical properties of nonlinear difference equations has been an area of intense interest in recent years (e.g., see [1-13]).

In [4], by analysis of semicycle structure, the authors discussed the global asymptotic stability of rational difference equation

$$
x_{n+1}=\frac{x_{n} x_{n-1}+1}{x_{n}+x_{n-1}}, \quad n=0,1, \ldots,
$$

where the initial values $x_{-1}, x_{0} \in(0,+\infty)$.

$\mathrm{Li}[5,6]$ investigated the qualitative behavior of the rational difference equations

$$
\begin{array}{r}
x_{n}=\frac{x_{n-1}+x_{n-2}+x_{n-4}+x_{n-1} x_{n-2} x_{n-4}+a}{1+x_{n-1} x_{n-2}+x_{n-2} x_{n-4}+x_{n-1} x_{n-4}+a}, \\
n=0,1,2, \ldots, \\
x_{n}=\frac{x_{n-2}+x_{n-3}+x_{n-4}+x_{n-2} x_{n-3} x_{n-4}+a}{1+x_{n-2} x_{n-3}+x_{n-3} x_{n-4}+x_{n-2} x_{n-4}+a}, \\
n=0,1,2, \ldots,
\end{array}
$$

with $x_{-4}, x_{-3}, \ldots, x_{-1} \in(0, \infty)$ and $a \in[0, \infty)$ via analysis of semicycle structure and verified that every solution of (2) converges to equilibrium 1 .
By using the transformation method, Berenhaut et al. [1] studied the behavior of positive solutions to the rational difference equation

$$
x_{n}=\frac{x_{n-k}+x_{n-m}}{1+x_{n-k} x_{n-m}}, \quad n=0,1,2, \ldots,
$$

with $x_{-m}, x_{-m+1}, \ldots, x_{-1} \in(0, \infty)$ and $1 \leq k<m$ and proved that every solution of (3) converges to the unique equilibrium 1. Based on the above facts, Berenhaut et al. [1] put forward the following two conjectures.

Conjecture 1. Suppose that $1 \leq k<l<m$ and that $\left\{x_{n}\right\}$ satisfies

$$
\begin{array}{r}
x_{n}=\frac{x_{n-k}+x_{n-l}+x_{n-m}+x_{n-k} x_{n-l} x_{n-m}}{1+x_{n-k} x_{n-l}+x_{n-l} x_{n-m}+x_{n-m} x_{n-k}}, \\
n=0,1,2, \ldots
\end{array}
$$

with $x_{-m}, x_{-m+1}, \ldots, x_{-1} \in(0, \infty)$. Then, the sequence $\left\{x_{n}\right\}$ converges to the unique equilibrium 1.

Conjecture 2. Suppose that $m$ is odd and $1 \leq k_{1}<k_{2}<\cdots<$ $k_{m}$, and define $S=\{1,2, \ldots, m\}$. If $\left\{x_{n}\right\}$ satisfies

$$
\begin{array}{r}
x_{n}=\frac{f_{1}\left(x_{n-k_{1}}, x_{n-k_{2}}, \ldots, x_{n-k_{m}}\right)}{f_{2}\left(x_{n-k_{1}}, x_{n-k_{2}}, \ldots, x_{n-k_{m}}\right)}, \\
n=0,1,2, \ldots,
\end{array}
$$


with $x_{-k_{m}}, x_{-k_{m}+1}, \ldots, x_{-1} \in(0, \infty)$, where

$$
\begin{aligned}
& f_{1}\left(x_{1}, x_{2}, \ldots, x_{m}\right) \\
& =\sum_{j \in\{1,3, \ldots, m\}} \sum_{\substack{\left\{t_{1}, t_{2}, \ldots, t_{j}\right\} \subset S ; \\
t_{1}<t_{2}<\cdots<t_{j}}} x_{t_{1}}, x_{t_{2}}, \ldots, x_{t_{j}}, \\
& f_{2}\left(x_{1}, x_{2}, \ldots, x_{m}\right) \\
& =1+\sum_{j \in\{2,4, \ldots, m-1\}} \sum_{\substack{\left\{t_{1}, t_{2}, \ldots, t_{j}\right\} \subset S ; \\
t_{1}<t_{2}<\cdots<t_{j}}} x_{t_{1}}, x_{t_{2}}, \ldots, x_{t_{j}},
\end{aligned}
$$

then the sequence $\left\{x_{n}\right\}$ converges to the unique equilibrium 1.

Recently, by method used in [4-6], the authors of [12] studied the global asymptotic stability of the following nonlinear difference equation.

$$
\begin{array}{r}
x_{n+1}=\frac{F\left(x_{n}, x_{n-1}, x_{n-2}, x_{n-3}\right)}{G\left(x_{n}, x_{n-1}, x_{n-2}, x_{n-3}\right)}, \\
n=0,1, \ldots,
\end{array}
$$

where

$$
\begin{aligned}
F(x, y, z, w)= & x^{\alpha_{1}} y^{\alpha_{2}}+x^{\alpha_{1}} z^{\alpha_{3}}+x^{\alpha_{1}} w^{\alpha_{4}}+y^{\alpha_{2}} z^{\alpha_{3}} \\
& +y^{\alpha_{2}} w^{\alpha_{4}}+z^{\alpha_{3}} w^{\alpha_{4}}+x^{\alpha_{1}} y^{\alpha_{2}} z^{\alpha_{3}} w^{\alpha_{4}}+1, \\
G(x, y, z, w)= & x^{\alpha_{1}}+y^{\alpha_{2}}+z^{\alpha_{3}}+w^{\alpha_{4}}+x^{\alpha_{1}} y^{\alpha_{2}} z^{\alpha_{3}} \\
& +x^{\alpha_{1}} y^{\alpha_{2}} w^{\alpha_{4}}+x^{\alpha_{1}} z^{\alpha_{3}} w^{\alpha_{4}}+y^{\alpha_{2}} z^{\alpha_{3}} w^{\alpha_{4}},
\end{aligned}
$$

the parameter $\alpha_{1} \in(0,1], \alpha_{2}, \alpha_{3}, \alpha_{4} \in(0,+\infty)$, and the initial values $x_{-3}, x_{-2}, x_{-1}, x_{0} \in(0,+\infty)$.

Motivated by the above studies, in this note, we propose and consider the following nonlinear difference equation.

$$
\begin{array}{r}
x_{n}=\frac{\prod_{i=1}^{v}\left(x_{n-k_{i}}^{\beta_{i}}+1\right)+\prod_{i=1}^{v}\left(x_{n-k_{i}}^{\beta_{i}}-1\right)}{\prod_{i=1}^{v}\left(x_{n-k_{i}}^{\beta_{i}}+1\right)-\prod_{i=1}^{v}\left(x_{n-k_{i}}^{\beta_{i}}-1\right)}, \\
n=0,1, \ldots,
\end{array}
$$

where $k_{i} \in \mathbb{N}(i=1,2, \ldots, v), v \geq 2, \beta_{1} \in[-1,1]$, $\beta_{2}, \beta_{3}, \ldots, \beta_{v} \in(-\infty,+\infty), x_{-m}, x_{-m+1}, \ldots, x_{-1} \in(0, \infty)$, and $m=\max _{1 \leq i \leq \nu}\left\{k_{i}\right\}$.

It is noticed that, letting $v=2, \beta_{1}=\beta_{2}=1, k_{1}=1$, and $k_{2}=2$, (9) reduces to (1); letting $v=3, \beta_{1}=\beta_{2}=\beta_{3}=$ $1, k_{1}=1, k_{2}=2$, and $k_{3}=4$ and $v=3, \beta_{1}=\beta_{2}=\beta_{3}=1$, $k_{1}=2, k_{2}=3$, and $k_{3}=4$, (9) reduces to (2); letting $v=$ 2, $\beta_{1}=\beta_{2}=1, k_{1}=k$, and $k_{2}=m$, (9) reduces to (3); letting $v=3, \beta_{1}=k, \beta_{2}=l$, and $\beta_{3}=m$, (9) reduces to (4); letting $v=4, \beta_{1} \in(0,1], \beta_{i}=\alpha_{i}(i=1,2,3,4), k_{1}=1, k_{2}=2$, $k_{3}=3$, and $k_{4}=4$, (9) reduces to (7); letting $v=m$ be odd, $1 \leq k_{1}<k_{2}<\cdots<k_{m}$, and $\beta_{1}=\beta_{2}=\cdots=\beta_{m}=1,(9)$ reduces to (5). Clearly, (5) is a special example of (9).

In 2007, Berenhaut and Stević [2] had proved Conjecture 1. In this paper, by making full use of analytical techniques, we mainly prove that the unique positive equilibrium point of (9) is globally asymptotically stable. It is clear that our result generalizes the corresponding works in $[1,2,4-9,12]$ and simultaneously conforms to Conjecture 2.

\section{Existence of a Unique Positive Equilibrium}

In this section, we mainly show the existence of a unique positive equilibrium of (9).

Theorem 3. In (9) there exists a unique positive equilibrium point $\bar{x}=1$.

Proof. A positive equilibrium point $\bar{x}$ of (9) satisfies the next equation:

$$
\bar{x}=\frac{\prod_{i=1}^{v}\left(\bar{x}^{\beta_{i}}+1\right)+\prod_{i=1}^{v}\left(\bar{x}^{\beta_{i}}-1\right)}{\prod_{i=1}^{v}\left(\bar{x}^{\beta_{i}}+1\right)-\prod_{i=1}^{v}\left(\bar{x}^{\beta_{i}}-1\right)},
$$

from which we may get

$$
(\bar{x}-1) \prod_{i=1}^{v}\left(\bar{x}^{\beta_{i}}+1\right)=(\bar{x}+1) \prod_{i=1}^{v}\left(\bar{x}^{\beta_{\mathrm{i}}}-1\right) ;
$$

that is,

$$
\begin{aligned}
& (\bar{x}-1)\left(\bar{x}^{\beta_{1}}+1\right) \prod_{i=2}^{v}\left(\bar{x}^{\beta_{i}}+1\right) \\
& =(\bar{x}+1)\left(\bar{x}^{\beta_{1}}-1\right) \prod_{i=2}^{v}\left(\bar{x}^{\beta_{i}}-1\right) .
\end{aligned}
$$

From the above equation, we can get

$$
\begin{aligned}
& \left(\bar{x}^{\beta_{1}+1}-1\right)\left(\prod_{i=2}^{v}\left(\bar{x}^{\beta_{i}}+1\right)-\prod_{i=2}^{v}\left(\bar{x}^{\beta_{i}}-1\right)\right) \\
& +\left(\bar{x}-\bar{x}^{\beta_{1}}\right)\left(\prod_{i=2}^{v}\left(\bar{x}^{\beta_{i}}+1\right)+\prod_{i=2}^{v}\left(\bar{x}^{\beta_{i}}-1\right)\right)=0 .
\end{aligned}
$$

One can see that for any $\bar{x}>0$ and $v \geq 2$,

$$
\begin{aligned}
& \prod_{i=2}^{v}\left(\bar{x}^{\beta_{i}}+1\right)-\prod_{i=2}^{v}\left(\bar{x}^{\beta_{i}}-1\right)>0, \\
& \prod_{i=2}^{v}\left(\bar{x}^{\beta_{i}}+1\right)+\prod_{i=2}^{v}\left(\bar{x}^{\beta_{i}}-1\right)>0 .
\end{aligned}
$$

(i) If $\beta_{1}=-1,0,1$, from (13) and (14), we can get that (9) has a unique positive equilibrium $\bar{x}=1$.

(ii) If $-1<\beta_{1}<0$ or $0<\beta_{1}<1$ and $0<\bar{x}<1$, we have

$$
\bar{x}<\bar{x}^{\beta_{1}}, \quad \bar{x}^{\beta_{1}+1}<1 .
$$

Further, we have

$$
\begin{aligned}
& \left(\bar{x}^{\beta_{1}+1}-1\right)\left(\prod_{i=2}^{v}\left(\bar{x}^{\beta_{i}}+1\right)-\prod_{i=2}^{v}\left(\bar{x}^{\beta_{i}}-1\right)\right) \\
& +\left(\bar{x}-\bar{x}^{\beta_{1}}\right)\left(\prod_{i=2}^{v}\left(\bar{x}^{\beta_{i}}+1\right)+\prod_{i=2}^{v}\left(\bar{x}^{\beta_{i}}-1\right)\right)<0 .
\end{aligned}
$$


(iii) If $-1<\beta_{1}<0$ or $0<\beta_{1}<1$ and $\bar{x}>1$, we have

$$
\begin{aligned}
& \left(\bar{x}^{\beta_{1}+1}-1\right)\left(\prod_{i=2}^{v}\left(\bar{x}^{\beta_{i}}+1\right)-\prod_{i=2}^{v}\left(\bar{x}^{\beta_{i}}-1\right)\right) \\
& +\left(\bar{x}-\bar{x}^{\beta_{1}}\right)\left(\prod_{i=2}^{v}\left(\bar{x}^{\beta_{i}}+1\right)+\prod_{i=2}^{v}\left(\bar{x}^{\beta_{i}}-1\right)\right)>0 .
\end{aligned}
$$

It is clear that (9) has a unique positive equilibrium $\bar{x}=1$. The proof is complete.

\section{Global Asymptotic Stability for the Unique Positive Equilibrium Point}

In this section, we give our main result.

Theorem 4. The unique positive equilibrium point $\bar{x}=1$ of (9) is globally asymptotically stable.

In order to prove Theorem 4, we introduce the following lemma by Kruse and Nesemann [3] and make full use of analytical techniques.

Lemma 5. Consider the difference equation

$$
x_{n+k}=f\left(x_{n+k-1}, \ldots, x_{n}\right), \quad n=0,1,2, \ldots,
$$

where $k \in \mathbb{N}$ and $f:(0, \infty)^{k} \rightarrow(0, \infty)$ is a continuous function with some unique equilibrium $\bar{x}$. Suppose that there is a $p \in \mathbb{N}$ such that for all solutions $\left\{x_{n}\right\}$ of (18)

$$
\left(x_{n}-x_{n+p}\right)\left(\frac{\bar{x}^{2}}{x_{n}}-x_{n+p}\right) \leq 0 \text {, }
$$

where equality holds if and only if $x_{n}=\bar{x}$. Then $\bar{x}$ is globally asymptotically stable.

Proof of Theorem 4. Let $\left\{x_{n}\right\}_{n=-m}^{\infty}$ be any solution of (9). We have

$$
\begin{aligned}
& x_{n}-x_{n-k_{1}}^{\beta_{1}} \\
& =\frac{\prod_{i=1}^{v}\left(x_{n-k_{i}}^{\beta_{i}}+1\right)+\prod_{i=1}^{v}\left(x_{n-k_{i}}^{\beta_{i}}-1\right)}{\prod_{i=1}^{v}\left(x_{n-k_{i}}^{\beta_{i}}+1\right)-\prod_{i=1}^{v}\left(x_{n-k_{i}}^{\beta_{i}}-1\right)}-x_{n-k_{1}}^{\beta_{1}} \\
& =\frac{\left(1-x_{n-k_{1}}^{\beta_{1}}\right) \prod_{i=1}^{v}\left(x_{n-k_{i}}^{\beta_{i}}+1\right)+\left(1+x_{n-k_{1}}^{\beta_{1}}\right) \prod_{i=1}^{v}\left(x_{n-k_{i}}^{\beta_{i}}-1\right)}{\prod_{i=1}^{v}\left(x_{n-k_{i}}^{\beta_{i}}+1\right)-\prod_{i=1}^{v}\left(x_{n-k_{i}}^{\beta_{i}}-1\right)} \\
& =\frac{\left(1-x_{n-k_{1}}^{\beta_{1}}\right)\left(1+x_{n-k_{1}}^{\beta_{1}}\right)\left(\prod_{i=2}^{v}\left(x_{n-k_{i}}^{\beta_{i}}+1\right)-\prod_{i=2}^{v}\left(x_{n-k_{i}}^{\beta_{i}}-1\right)\right)}{\prod_{i=1}^{v}\left(x_{n-k_{i}}^{\beta_{i}}+1\right)-\prod_{i=1}^{v}\left(x_{n-k_{i}}^{\beta_{i}}-1\right)} \quad n=0,1, \ldots,
\end{aligned}
$$

$$
\begin{aligned}
& \frac{1}{x_{n-k_{1}}^{\beta_{1}}}-x_{n} \\
= & \frac{1}{x_{n-k_{1}}^{\beta_{1}}}-\frac{\prod_{i=1}^{v}\left(x_{n-k_{i}}^{\beta_{i}}+1\right)+\prod_{i=1}^{v}\left(x_{n-k_{i}}^{\beta_{i}}-1\right)}{\prod_{i=1}^{v}\left(x_{n-k_{i}}^{\beta_{i}}+1\right)-\prod_{i=1}^{v}\left(x_{n-k_{i}}^{\beta_{i}}-1\right)} \\
= & \frac{\left(1-x_{n-k_{1}}^{\beta_{1}}\right) \prod_{i=1}^{v}\left(x_{n-k_{i}}^{\beta_{i}}+1\right)-\left(1+x_{n-k_{1}}^{\beta_{1}}\right) \prod_{i=1}^{v}\left(x_{n-k_{i}}^{\beta_{i}}-1\right)}{\prod_{i=1}^{v}\left(x_{n-k_{i}}^{\beta_{i}}+1\right)-\prod_{i=1}^{v}\left(x_{n-k_{i}}^{\beta_{i}}-1\right)}, \\
= & \frac{\left(1-x_{n-k_{1}}^{\beta_{1}}\right)\left(1+x_{n-k_{1}}^{\beta_{1}}\right)\left(\prod_{i=2}^{v}\left(x_{n-k_{i}}^{\beta_{i}}+1\right)+\prod_{i=2}^{v}\left(x_{n-k_{i}}^{\beta_{i}}-1\right)\right)}{\prod_{i=1}^{v}\left(x_{n-k_{i}}^{\beta_{i}}+1\right)-\prod_{i=1}^{v}\left(x_{n-k_{i}}^{\beta_{i}}-1\right)},
\end{aligned}
$$

It follows from (20) that

$$
\begin{aligned}
& x_{n+k_{1}}-x_{n}^{\beta_{1}} \\
& =\frac{\left(1-x_{n}^{\beta_{1}}\right)\left(1+x_{n}^{\beta_{1}}\right)\left(\prod_{i=2}^{v}\left(x_{n+k_{1}-k_{i}}^{\beta_{i}}+1\right)-\prod_{i=2}^{v}\left(x_{n+k_{1}-k_{i}}^{\beta_{i}}-1\right)\right)}{\prod_{i=1}^{v}\left(x_{n+k_{1}-k_{i}}^{\beta_{i}}+1\right)-\prod_{i=1}^{v}\left(x_{n+k_{1}-k_{i}}^{\beta_{i}}-1\right)} \\
& n=-k_{1},-k_{1}+1, \ldots, \\
& \begin{array}{c}
\frac{1}{x_{n}^{\beta_{1}}}-x_{n+k_{1}} \\
\prod_{i=1}^{v}\left(x_{n+k_{1}-k_{i}}^{\beta_{i}}+1\right)-\prod_{i=1}^{v}\left(x_{n+k_{1}-k_{i}}^{\beta_{i}}-1\right) \\
n=-k_{1},-k_{1}+1, \ldots .
\end{array}
\end{aligned}
$$

Clearly, from (21), we have

$$
\begin{array}{r}
\left(x_{n}^{\beta_{1}}-x_{n+k_{1}}\right)\left(\frac{1}{x_{n}^{\beta_{1}}}-x_{n+k_{1}}\right) \leq 0, \\
n=-k_{1},-k_{1}+1, \ldots
\end{array}
$$

From (22), we have

$$
\begin{array}{r}
1-x_{n+k_{1}}\left(\frac{1}{x_{n}^{\beta_{1}}}+x_{n}^{\beta_{1}}\right)+x_{n+k_{1}}^{2} \leq 0, \\
n=-k_{1},-k_{1}+1, \ldots
\end{array}
$$

If $\beta_{1}= \pm 1$, it is clear that

$$
\frac{1}{x_{n}^{\beta_{1}}}+x_{n}^{\beta_{1}}=\frac{1}{x_{n}}+x_{n}
$$

If $0<x_{n}<1$ and $-1<\beta_{1}<1$, we have $x_{n}<x_{n}^{\beta_{1}}$ and $0<x_{n}^{\beta_{1}+1}<1$, so that

$$
\left(x_{n}-x_{n}^{\beta_{1}}\right)\left(1-\frac{1}{x_{n} x_{n}^{\beta_{1}}}\right)>0 .
$$


Similarly, if $x_{n}>1$ and $-1<\beta_{1}<1$, we have $x_{n}>x_{n}^{\beta_{1}}$ and $x_{n}^{\beta_{1}+1}>1$, so that

$$
\left(x_{n}-x_{n}^{\beta_{1}}\right)\left(1-\frac{1}{x_{n} x_{n}^{\beta_{1}}}\right)>0 .
$$

Hence, for $-1 \leq \beta_{1} \leq 1$, we always have

$$
\frac{1}{x_{n}^{\beta_{1}}}+x_{n}^{\beta_{1}} \leq \frac{1}{x_{n}}+x_{n} .
$$

Further, from (23) and (27), we have

$$
\begin{array}{r}
1-x_{n+k_{1}}\left(\frac{1}{x_{n}}+x_{n}\right)+x_{n+k_{1}}^{2} \\
\leq 1-x_{n+k_{1}}\left(\frac{1}{x_{n}^{\beta_{1}}}+x_{n}^{\beta_{1}}\right)+x_{n+k_{1}}^{2} \leq 0, \\
n=-k_{1},-k_{1}+1, \ldots
\end{array}
$$

Therefore,

$$
\begin{array}{r}
\left(x_{n}-x_{n+k_{1}}\right)\left(\frac{1}{x_{n}}-x_{n+k_{1}}\right) \leq 0 \\
n=-k_{1},-k_{1}+1, \ldots,
\end{array}
$$

where equality holds if and only if $x_{n}=\bar{x}=1$. By Lemma 5 and (29), with $p=k_{1} \in \mathbb{N}$, it follows that the unique positive equilibrium point $\bar{x}=1$ of (9) is globally asymptotically stable. The proof is complete.

\section{Conflict of Interests}

The author declares that there is no conflict of interests regarding the publication of this paper.

\section{Acknowledgments}

The author is grateful to the referees for their careful reading of the paper and many valuable comments and suggestions that greatly improved the presentation of this work. This paper is supported partly by Hunan Provincial Natural Science Foundation of China (no. 13JJ3075), Soft Science Fund of Science and Technology Department of Hunan Province (no. 2011ZK3066), Start-up Fund of University of South China (no. 2011XQD49), and the construct program in USC.

\section{References}

[1] K. S. Berenhaut, J. D. Foley, and S. Stević, "The global attractivity of the rational difference equation $y_{n}=y_{n-k}+y_{n-m} / 1+$ $y_{n-k} y_{n-m}$ " Applied Mathematics Letters, vol. 20, no. 1, pp. 54-58, 2007.

[2] K. S. Berenhaut and S. Stević, "The global attractivity of a higher order rational difference equation," Journal of Mathematical Analysis and Applications, vol. 326, no. 2, pp. 940-944, 2007.

[3] N. Kruse and T. Nesemann, "Global asymptotic stability in some discrete dynamical systems," Journal of Mathematical Analysis and Applications, vol. 235, no. 1, pp. 151-158, 1999.
[4] X. Li and D. Zhu, "Global asymptotic stability in a rational equation," Journal of Difference Equations and Applications, vol. 9, no. 9, pp. 833-839, 2003.

[5] X. Li, "Qualitative properties for a fourth-order rational difference equation," Journal of Mathematical Analysis and Applications, vol. 311, no. 1, pp. 103-111, 2005.

[6] X. Li, "Global behavior for a fourth-order rational difference equation," Journal of Mathematical Analysis and Applications, vol. 312, no. 2, pp. 555-563, 2005.

[7] T. Sun and H. Xi, "Global asymptotic stability of a higher order rational difference equation," Journal of Mathematical Analysis and Applications, vol. 330, no. 1, pp. 462-466, 2007.

[8] T. Sun and H. Xi, "Global attractivity for a family of nonlinear difference equations," Applied Mathematics Letters, vol. 20, no. 7, pp. 741-745, 2007.

[9] T. Sun, H. Xi, and C. Han, "Stability of solutions for a family of nonlinear difference equations," Advances in Difference Equations, vol. 2008, Article ID 238068, 6 pages, 2008.

[10] X. Yang, D. J. Evans, and G. M. Megson, "Global asymptotic stability in a class of Putnam-type equations," Nonlinear Analysis, Theory, Methods and Applications, vol. 64, no. 1, pp. 42-50, 2006.

[11] X. Yang, Y. Y. Tang, and J. Cao, "Global asymptotic stability of a family of difference equations," Computers and Mathematics with Applications, vol. 56, no. 10, pp. 2643-2649, 2008.

[12] D. Li, P. Li, and X. Li, "Dynamical properties for a class of fourth-order nonlinear difference equations," Advances in Difference Equations, vol. 2008, Article ID 678402, 13 pages, 2008.

[13] M. Liao, X. Tang, and C. Xu, "On the rational difference equation $x_{n}=1+\left(1-x_{n-k}\right)\left(1-x_{n-l}\right)\left(1-x_{n-m}\right) /\left(x_{n-k}+x_{n-l}+x_{n-m}\right), "$ Journal of Applied Mathematics and Computing, vol. 35, no. 1-2, pp. 63-71, 2011. 


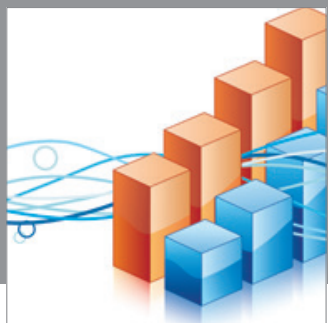

Advances in

Operations Research

mansans

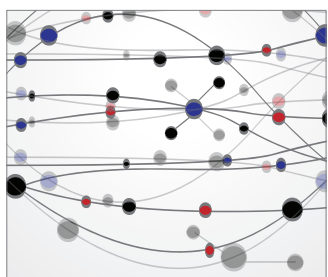

The Scientific World Journal
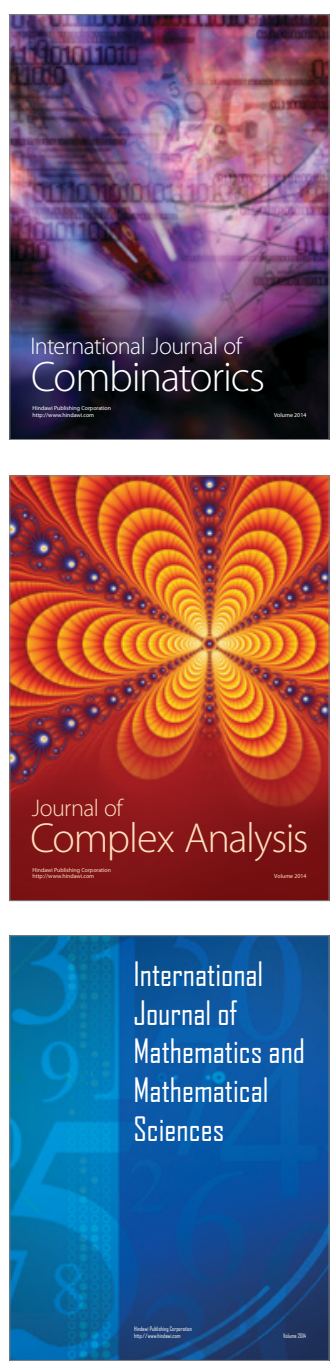
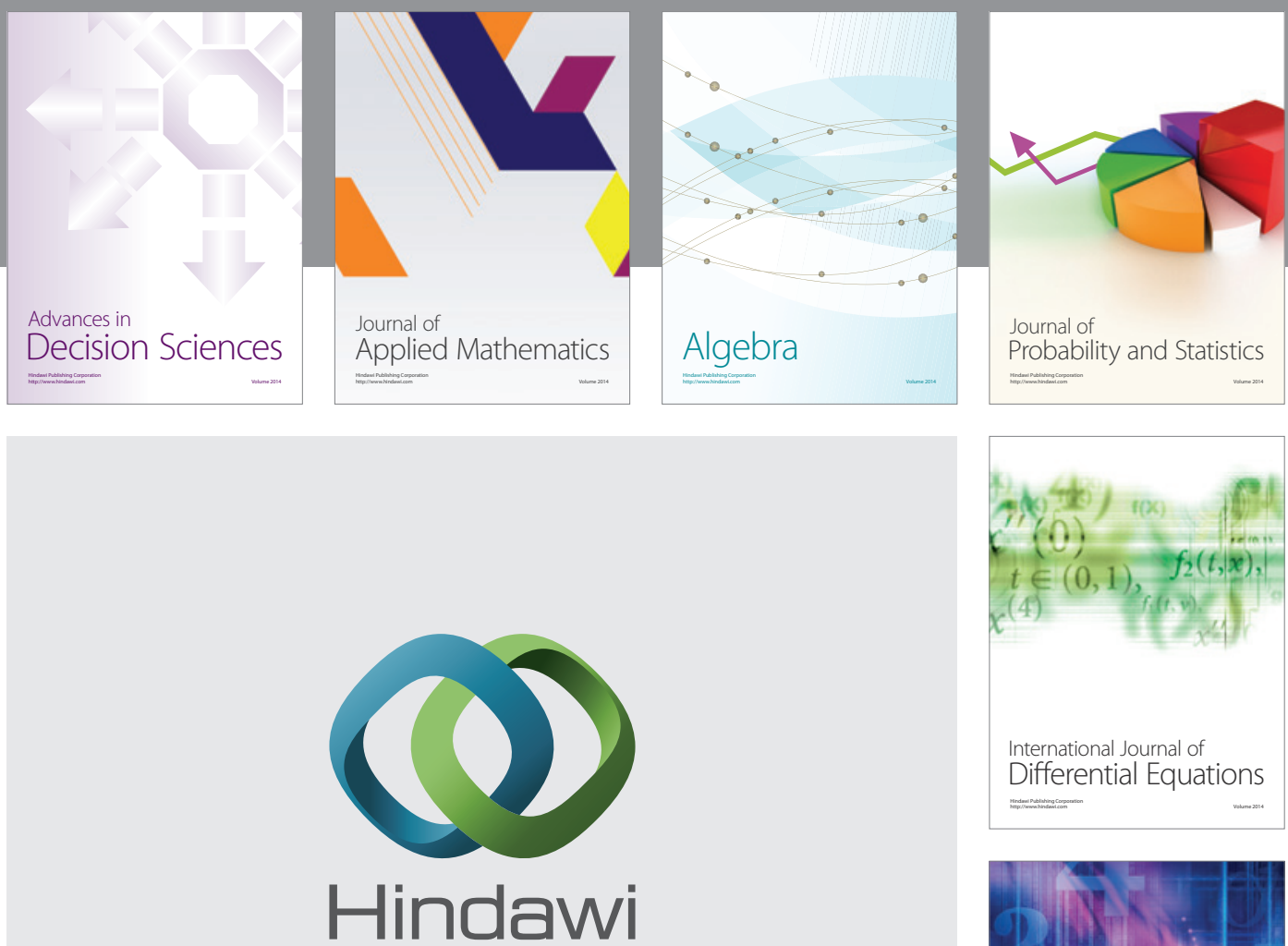

Submit your manuscripts at http://www.hindawi.com
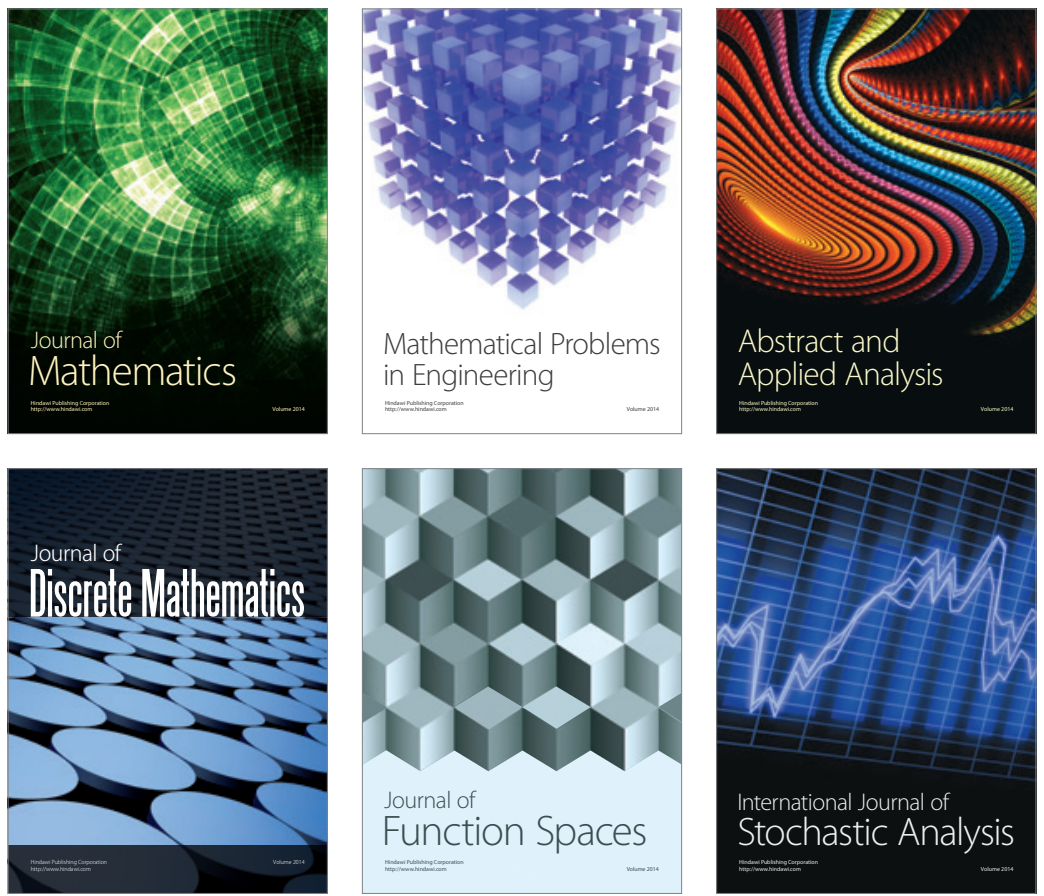

Journal of

Function Spaces

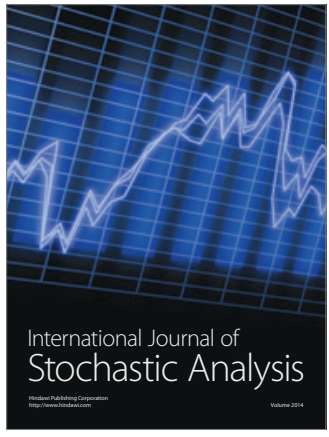

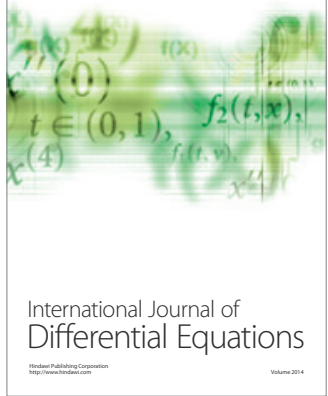
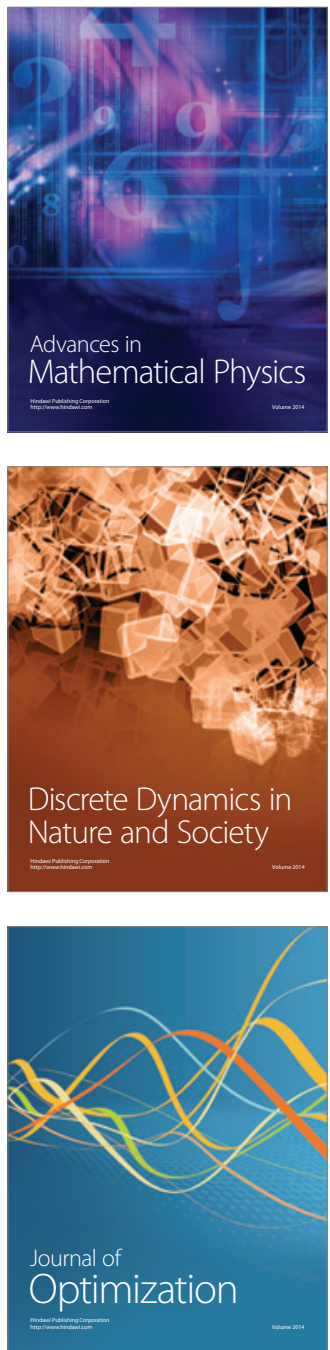